\title{
Perspective
}

PERSPECTIVE Actualité en histoire de l'art

$2 \mid 2020$

Danser

\section{Danser l'identité nationale espagnole à Paris et à New York (1928-1930) : Antonia Mercé, La Argentina, entre néo-classicisme, modernisme et expression populaire}

The Dance of Spanish National Identity in Paris and New York (1928-1930):

Antonia Mercé, La Argentina, between Neoclassicism, Modernism and the Folk

In Paris und New York die spanische Nationalidentität tanzen (1928-1930).

Antonia Mercé, La Argentina, zwischen Neoklassizimus, Moderne und

Volkstümlichkeit

Danzare l'identità nazionale spagnola a Parigi e a New York (1928-1930): Antonia Mercé, La Argentina, tra neoclassicismo, modernismo ed espressione popolare Bailar la identidad nacional española en Paris y en Nueva York (1928-1930): Antonia Mercé, La Argentina, entre neoclasicismo, modernismo y expresión popular

\section{Mark Franko}

Traducteur : François Boisivon

\section{(e) OpenEdition}

\section{Journals}

\section{Édition électronique}

URL : https://journals.openedition.org/perspective/21232

DOI : 10.4000/perspective.21232

ISSN : 2269-7721

Éditeur

Institut national d'histoire de l'art

\section{Édition imprimée}

Date de publication : 30 décembre 2020

Pagination : 207-218

ISBN : 978-2-917902-90-5

ISSN : $1777-7852$

Référence électronique

Mark Franko, « Danser l'identité nationale espagnole à Paris et à New York (1928-1930) : Antonia Mercé, La Argentina, entre néo-classicisme, modernisme et expression populaire », Perspective [En ligne], 2 | 2020, mis en ligne le 30 juin 2021, consulté le 30 juillet 2022. URL : http:// journals.openedition.org/perspective/21232; DOI : https://doi.org/10.4000/perspective.21232 
Tous droits réservés 


\title{
Danser l'identité nationale espagnole à Paris et à New York (1928-1930) :
}

\author{
Antonia Mercé, La Argentina, \\ entre néo-classicisme, modernisme \\ et expression populaire
}

\author{
Mark Franko
}

Les chercheurs qui s'intéressent à la danse connaissent bien le phénomène : durant la première moitié du $\mathrm{XX}^{\mathrm{e}}$ siècle, la danse et l'identité nationale furent pensées comme se reflétant l'une l'autre, que l'on considérât les performances des solistes de la danse moderne, celles des compagnies de ballet ou de celles qui se spécialisaient dans ce qu'on appelait les "danses exotiques "-plus tard, " ethniques "-, portées à la scène. Dans ce large éventail de modalités artistiques possibles par lequel la danse s'est définie comme un art moderne, il n'est pas sans intérêt de rappeler que l'idée de la danse comme incarnation en mouvement du caractère national vaut aussi bien pour l'invention des nouveaux vocabulaires de la danse moderne (ceux que créèrent Mary Wigman, Martha Graham ou Michio Ito, pour ne citer que ces trois noms emblématiques) que pour les conceptions académiques de la danse de ballet (qu'il s'agisse des Ballets suédois, des Ballets russes ou de l'Opéra de Paris). Mais cette idée marque aussi les représentations de l'identité ethnique données par des danses traditionnelles censées n'avoir ni ambitions modernistes, ni fondements académiques (ainsi des spectacles de La Meri, de Zora Neale Hurston, d'Uday Shankar ou d'autres). L'adaptation pour la scène nécessitait souvent une stylisation ou une modernisation personnelle des matériaux d'origine découverts par la recherche ethnographique. En réalité, nombre de ces projets se chevauchaient, de sorte qu'une danseuse comme La Argentina, qui avait reçu une formation classique, pouvait entreprendre une recherche ethnographique et produire en même temps des solos spectaculaires ou des ballets complets pour sa compagnie de danse ${ }^{1}$. En outre, le sens que l'on attribuait à l'identité nationale incarnée variait vraisemblablement en fonction du lieu où les artistes étaient invités à présenter leur travail et de leur réception à l'étranger par les critiques ou les intellectuels - eux-mêmes étant souvent des personnes déplacées. Depuis que la recherche sur la danse s'intéresse aux danseurs et aux compagnies qui traversèrent les frontières nationales - et par conséquent aux échanges internationaux plus qu'à la nationalité des danseurs en elle-même -, l'interprétation des influences réciproques du mouvement dansé et de l'identité nationale est devenue une tâche beaucoup plus complexe. 
La Argentina (fig. 1) a commencé de se produire en France à Paris, en 1910, et c'est à partir de 1921 que s'y tiennent ses grandes représentations, à l'Opéra de Paris et à l'Opéra comique, avec les Ballets espagnols, la compagnie qu'elle a créée ${ }^{2}$. Mais elle a également entrepris, tout au long des années 1920, des tournées dans le monde entier, qui lui permirent de s'affirmer comme une star internationale avec ses solos de danse espagnole. Cet article se penche sur deux exemples de sa réception critique : à Paris, en 1928, sous la plume d'André Levinson (1887-1933), Russe expatrié, critique de danse et historien, et à New York, en 1930, par le philosophe espagnol Federico de Onís ${ }^{3}$ (1888-1966). J'y analyse la façon dont les deux critiques interprétèrent son nationalisme et les liens de leur réception avec une perception du folklore comme élément national du " classique " (procédant donc du " colonisant "), alors même que les matériaux ethnographiques rassemblés par La Argentina témoignent de la nature colonisée de l'identité espagnole. Suivant cette voie, je poursuis et j'étends, dans cet article, mon analyse des tensions entre l'esthétique moderniste, le néo-classicisme et l'esthétique folklorique dans la danse, entre les deux guerres, en France, que je tente ici de penser à partir de l'exemple de l'artiste espagnole ${ }^{4}$.

\section{Les atours de la danse moderne}

Le ballet occupe, par rapport aux autres arts, une position singulière dans le contexte du néo-classicisme de l'entre-deux-guerres. Ainsi, le tournant vers le néo-classicisme des arts visuels en France, dans les années 1920, est-il considéré comme un rejet du modernisme ${ }^{5}$; mais dans le ballet, le tournant néo-classique fut en réalité la condition même de l'ouverture des ballets au modernisme - sur fond, cependant, de revendications concurrentes émanant du champ naissant de l'ethnographie française quant à l'importance de la danse folklorique, incarnation de l'identité nationale, profondément ancrée dans l'histoire malgré son divorce d'avec les traditions académiques au XVII siècle. On assista également, dans les années 1930, à une revendication de la modernité de la danse folklorique, dans la mesure où ses traditions étaient considérées comme promises à un renouvellement. Les thèmes folkloriques étaient par ailleurs présents dans le ballet d'avant-garde, illustrés notamment par Jean Cocteau et Rolf de Maré. Dans le cas de La Argentina, les mêmes éléments entrent en jeu dans son évocation aussi bien de l'école du boléro que des apports folkloriques de la danse espagnole, ou dans son usage du flamenco.

L'un des principaux architectes de la réception de La Argentina en France fut André Levinson, expatrié russe, historien et critique de danse, qui devint le principal défenseur des chorégraphies de Serge Lifar à l'Opéra de Paris dans les années 1930, qu'il applaudit parce qu'elles marquaient pour lui un retour aux traditions classiques de la danse académique française. Émigré en France après la révolution russe, Levinson s'intéressait surtout au ballet dans la tradition française. À travers l'exemple de La Argentina dans les années 1920, on peut voir comment ses conceptions de la danse et de l'identité nationale s'appliquent, avant l'ascension de Serge Lifar, à une artiste qui n'est pas française. En 1928, Levinson publie en anglais un livre consacré à Antonia Mercé, où il esquisse la théorie d'une identité nationale espagnole dans la danse. La contribution d'Onís à un ouvrage dédié à la danseuse, en 1930, après ses représentations à New York, témoigne d'une autre réception, plus proprement espagnole, mais provenant elle aussi d'un expatrié. Onís avait quitté en 1916 l'université de Salamanque pour New York. Il avait obtenu un poste de chercheur à la Columbia University, où il fonda en 1930 une " maison de l'Espagne ", la Casa Hispánica, et développa l'étude de la culture hispanique, notamment au niveau de 
l'enseignement supérieur ${ }^{6}$. Levinson et Onís impriment à la réception de La Argentina des points de vue très différents, tant sur le plan culturel que disciplinaire. Levinson était le principal critique de danse à Paris dans l'entre-deux-guerres, tandis qu'Onís était un philosophe espagnol, spécialiste de littérature, influencé par Miguel de Unamuno et José Ortega y Gasset. L'un et l'autre reconnaissent l'utilisation que fait la danseuse des traditions nationales de la danse espagnole, mais ils ne comprennent pas du tout de la même manière ce que son usage particulier de ces traditions incarne de l'idée d'un nationalisme espagnol. Ce que partagent les deux auteurs, c'est une reconnaissance de la singularité de La Argentina, qui l'autorise à parler tant au monde de la danse qu'à ceux de la littérature et de la philosophie. En examinant cette question du point de vue du langage critique, je serai amené à proposer une construction de l'histoire de la danse à partir de l'analyse rhétorique de cette critique.

Si l'interprétation du nationalisme que donne Levinson se développe dans le contexte français du néo-classicisme, en lien étroit avec l'académisme, Onís semble quant à

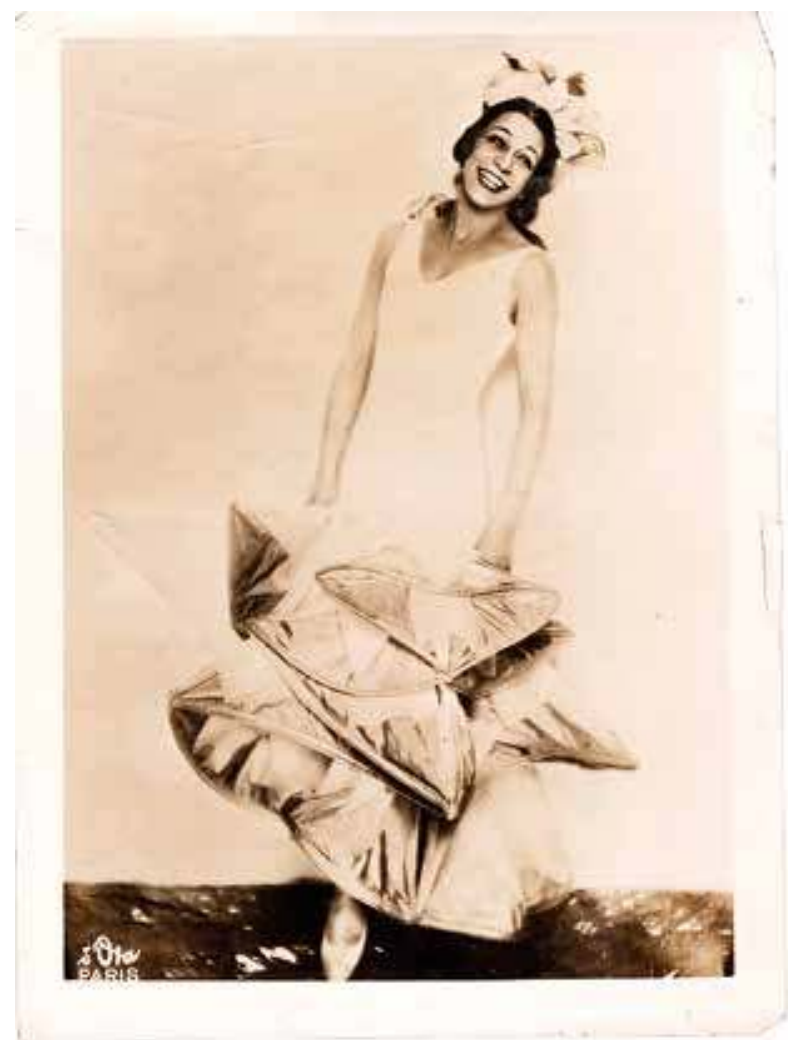

1. Dora Kallmus, dite Madame d'Ora, portrait d'Antonia Mercé, La Argentina, dans un costume de Néstor de la Torre, pour Triana (Isaac Albéniz), vers 1929, collection privée. lui influencé par le regeneracionismo et l'idée qu'exprimait Ortega y Gasset d'une identité espagnole à inventer ${ }^{7}$. Il faut aussi mentionner, dans ce contexte, l'article de Paul Valéry intitulé " Philosophie de la danse ", lu par le poète lors d'une représentation donnée par " $\mathrm{M}^{\mathrm{me}}$ Argentina », à Paris, en 1936. J'ai commenté ailleurs ce texte, à la lumière du ballet de Kazuo Ohno, Admiring La Argentina ${ }^{8}$. Dans cette allocution, Valéry présente La Argentina comme la créatrice d'un temps et d'un espace autonomes au sein desquels survient la danse - idée que l'on pourrait, en un certain sens, lire comme liée à l'urgence de concevoir l'identité au présent. Le cadre temporel et spatial abstrait où s'inscrit le regard que le poète porte sur la danse laisse peu de place, en revanche, aux connotations identitaires qui sous-tendent l'accueil fait à la danseuse par Levinson et Onís.

\section{L'identité nationale espagnole analysée dans son mouvement}

Chez ces deux auteurs, j’examinerai la rhétorique déployée dans leur tentative de définir la danse de La Argentina comme la quintessence du style espagnol pour l'un, ou comme expression personnelle et transitoire de l'identité nationale espagnole pour l'autre9. Chez l'un comme chez l'autre, il ne fait aucun doute que la danse de La Argentina représente d'abord l'identité nationale espagnole, mais c'est précisément ce qui contraint Levinson à 


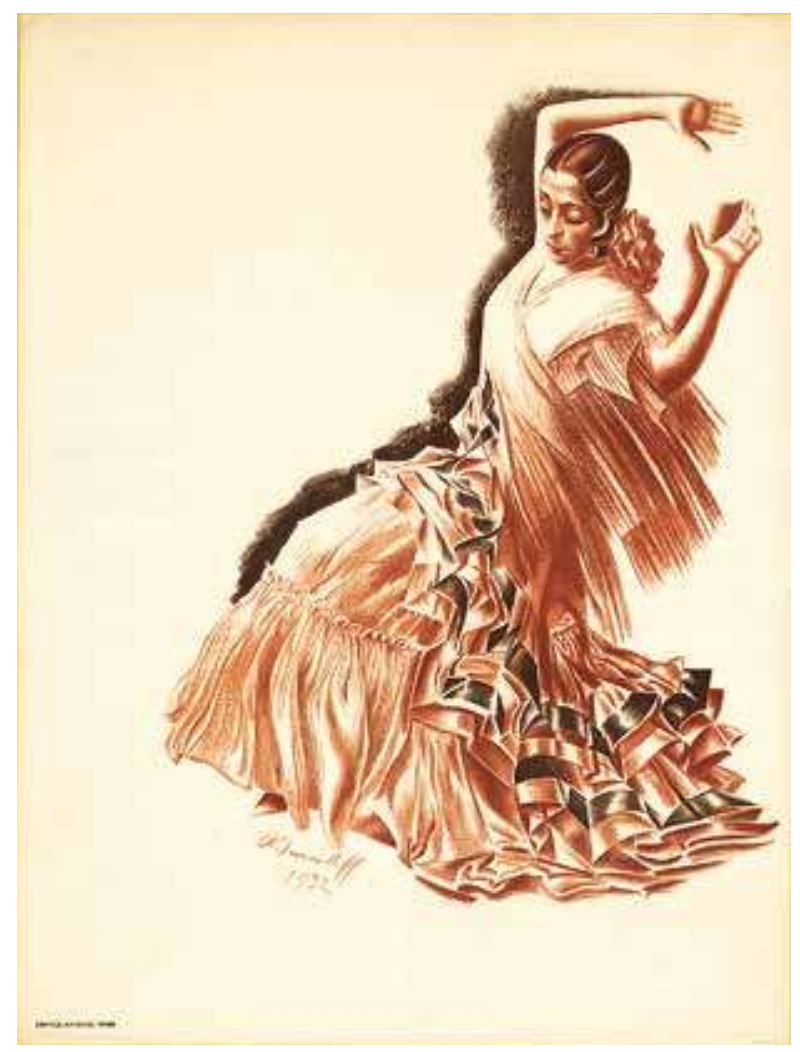

2. Aleksandr Evgen'evič Âkovlev, [La Argentina, affiche sans lettre], Paris, France-Affiches, 1932, Paris, Bibliothèque nationale de France, département Estampes et Photographie.

3. Dora Kallmus, dite Madame d'Ora, carte postale avec un montage de plusieurs portraits de La Argentina, 1930, Paris, Bibliothèque nationale de France, département Musique. calquer sur le modèle français le classicisme de la danseuse. Sa conception est orientaliste ${ }^{10}$. Il est particulièrement intéressant de suivre la logique de Levinson dans son étude d'un art populaire classique en Espagne, qu'il ramène au néo-classicisme, alors même que ce dernier, du moins dans ses manifestations les plus officielles, telles qu'on peut les associer à l'Opéra de Paris, se tient habituellement dans l'ignorance revendiquée des influences vernaculaires. Sa démarche part de l'hypothèse qu'une danse exprimant l'identité nationale est nécessairement enracinée dans des principes académiques classiques. Ces principes, dans le cas de La Argentina, puisent à différentes sources : l'école du boléro, le flamenco et les danses folkloriques et régionales. Tous éléments que Levinson place sous l'égide d'un classicisme résultant du renouvellement même, par la danseuse, de formes qui s'étaient discréditées en sombrant dans l'emphase. Levinson s'appuie sur l'idée du triomphe de la forme, idée intrinsèque, comme je l'ai montré ailleurs, au ballet néo-classique. Le problème de la variété des styles et des vocabulaires auxquels recourt La Argentina ne peut pourtant être résolu par les principes formels d'une unique danse " classique " - le boléro - dont l'origine remonte au XVIII ${ }^{\mathrm{e}}$ siècle. Pour cette raison, il est plus complexe de situer dans le temps le classicisme de La Argentina que le néoclassicisme des ballets français, du moins tel que le conçoit Levinson ${ }^{11}$.

Un autre plan de l'analyse de Levinson s'attache au phénomène de la modernisation des sources de la danseuse. Les mouvements que crée La Argentina tracent des lignes dans l'espace et laissent le souvenir de formes singulières qui sont indubitablement modernes, comme l'attestent les nombreuses tentatives de saisir son mouvement dans des croquis ou des caricatures prises sur le vif (fig. 2 et $\mathbf{3}$ ). Doter le geste de lignes relève d'une démarche moderniste et, lorsque des matériaux populaires sont stylisés au point de mériter leur entrée dans la catégorie de l'art comme expression universelle, la trace qui en résulte peut être considérée comme " classique ", c'est-à-dire comme représentative des caractéristiques essentielles de ce qu'elle décrit. Afin de poser l'esthétique modernisée du populaire comme expression de l'esprit national de l'Espagne, Levinson se devait pourtant de donner au matériau que travaillait La Argentina une acception classique, à double titre : qu'on puisse tout d'abord le saisir en termes formels, stylistiquement, comme autonome ; deuxièmement, puisqu'il provenait du peuple, qu'il puise à la source inépuisable et anonyme d'un mouvement qui pouvait être identifié à l'esprit national ou, pour reprendre les termes de l'époque, à " l'esprit de la race ». Jusqu'à un certain 


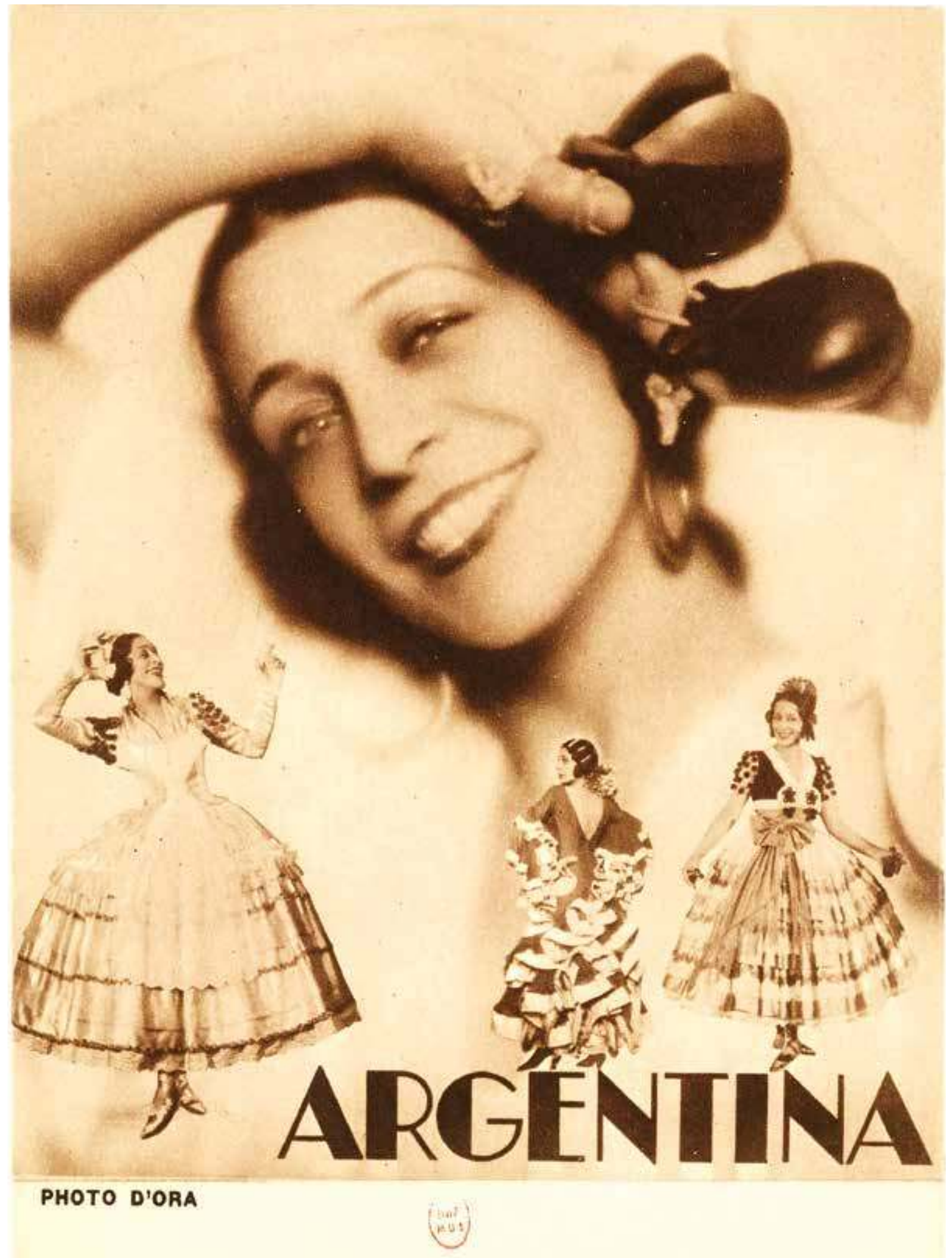


point, c'était ce que La Argentina opérait elle-même avec ses sources populaires, comme elle l'a expliqué : " J'ai tenté de réunir deux danses, la danse espagnole et ce qu'on appelle la danse "moderne"12. "Idoia Murga Castro analyse les types de références aux danses indigènes qui conditionnèrent la réception globale du travail de Mercé en tant que représentation exotique de l'hispanité. Mais dans les écrits de Levinson sur la star, au-delà des connotations péjoratives concernant l'exotisme, on assiste aussi à la convergence, dans la singularité d'un corps dansant, du moderne, du classique et du populaire. Ces empreintes différentes, quoique simultanées, sont en quelque sorte maintenues ensemble par l'enveloppe du national.

Si l'importance de la danse vernaculaire pour le classicisme du ballet a été défendue dans les années 1920 par Rolf de Maré et Jean Cocteau, son discours sur La Argentina constitue l'unique contribution de Levinson à ce courant de pensée ${ }^{13}$. L'idée d'une expression populaire devenue en elle-même constitutive du " classique ", une fois filtrée par le formalisme moderniste, offre une variation intéressante de sa théorie du ballet néoclassique français. La façon dont Levinson se sort de l'impasse d'une identité proprement nationale, qu'on pourrait considérer comme exotique, me semble ici particulièrement intéressante. Il n'échappe malheureusement pas à certaines conceptions racistes du ballet dans ses rapprochements avec les danses afro-américaines. Ma comparaison de ces deux réceptions critiques de La Argentina accorde donc une attention particulière au terme " classique " pour qualifier des représentations dansées de l'entre-deux-guerres qui étaient indubitablement modernistes, mais qui puisaient aussi à des recherches portant sur les formes folkloriques. Pourquoi la liaison de ces termes convoque-t-elle le national - sans parler du racial ? En un certain sens, nous nous situons ici à un point de convergence des théories raciales, des études ethniques et de la danse pour ce qui concerne la première moitié du XX $\mathrm{XX}^{\mathrm{e}}$ siècle. Même dans la danse la plus avant-gardiste présentée sur scène dans l'entre-deux-guerres, cet environnement semble inévitable et les termes en sont, dans une certaine mesure, interchangeables ${ }^{14}$.

La seconde conception, celle d'Onís, évoque l'individualisme irréductible et la subjectivité de la danseuse moderne, qui invente un langage personnel inimitable en vertu duquel sa danse devient l'expression d'une identité nationale. L'identité est alors définie par un geste singulier, par nature plus anthropologique car il ne nous ramène pas nécessairement à l'idée d'une essence consubstantielle à la typologie raciale. Tels sont, dans un certain sens, les pôles apollinien et dionysiaque du modernisme en danse, si souvent évoqué dans la première moitié du $\mathrm{XX}^{\mathrm{e}}$ siècle $^{15}$.

La question d'une réception critique duale de La Argentina, danseuse déplacée, qui travaillait entre l'Argentine - son pays natal -, l'Espagne - le lieu de sa tradition - et Paris - où elle vivait et travaillait - par deux critiques émigrés, par conséquent euxmêmes déplacés - Levinson venu d'URSS à Paris et Onís, d'Espagne (hésitante entre monarchie parlementaire et dictature militaire) à New York - met en évidence la question de l'espace dans lequel survient la danse. La danse prend place dans un espace qui est lui-même géographiquement déplacé au moment même où l'identité nationale essentialisée devient sa représentation critique la plus marquante. En d'autres termes, la danse est exilée, de sorte que les espaces et les cultures dont elle se réclame sont imaginaires, car déterminés par les traditions critiques de l'espace au sein duquel survient le mouvement. Les mouvements ne sont pas ancrés dans le sol sur lequel ils sont dansés. À Paris, la critique appartient à un contexte où le statut classique du ballet français est particulièrement important, et par conséquent à un espace où la notion de " classique " est dans une grande mesure informée par la tradition française. À New York, l'espace, tel qu'Onís mais aussi Lorca en font l'expérience, est un espace aliéné, habité par le masque 
de la condition noire, qui imprègne la description que Lorca donne de New York dans les poèmes qu'il écrit lors de son séjour à la Columbia University en 1929-1930; un espace, par conséquent, où le mouvement peut être lu comme extrêmement personnel, dans une relation à la mécanisation et à la modernité considérées comme des forces négatives. Ce que révèle cette étude comparative, c'est que la notion même d'identité nationale, dans les années 1928-1930, se trouve radicalement questionnée, car elle est pour Levinson monumentale et formelle tandis que, pour Onís, elle est éphémère, fondée sur une inflexion rythmique.

Levinson apprécie la spontanéité apparente de la représentation donnée par La Argentina, mais il est convaincu, en dernière analyse, que tout ce qu'elle fait est le résultat d'un art calculé. " Elle capture le tumulte intérieur pour le réduire à une forme. Elle manie ses émotions avec la même sagacité qu'un chimiste manie un explosif ${ }^{16}$. " Si c'est précisément parce qu'il renonce à une expression individuelle vraiment singulière, qui ne pourrait être imaginée en elle-même comme une permanence transmissible à d'autres, que ce formalisme est adapté à l'expression d'une identité nationale, le nationalisme espagnol est aussi, pour Levinson, un matériau potentiellement exotique dans la mesure où, en France, il est reçu comme tel. Les commentateurs conservateurs considéraient comme éminemment rationnelle l'expression esthétique de l'identité nationale française, alors que l'identité nationale espagnole leur apparaissait comme émotionnellement turbulente. Ce qui me semble particulièrement intéressant dans le discours de Levinson, c'est que dès lors que La Argentina accède à l'incarnation de l'identité nationale pour ce qui concerne l'Espagne, cette identité ne peut plus être reçue comme étant pleinement exotique, car elle appartient désormais à une conscience classique et classicisante de l'identité. De même, c'est à la fois comme colonisante et colonisée que Levinson comprend la gestuelle espagnole. Par conséquent, mon analyse de sa rhétorique se concentre sur ce problème et sur la manière particulière dont il le résout. La question du nationalisme espagnol, dont le classicisme est pour Levinson le fruit d'une lutte, est plus complexe à ses yeux que celle du nationalisme français. Si le critique a salué l'expression classique de l'identité nationale espagnole par la danseuse, il la conçoit en même temps comme subalterne eu égard à l'identité française. L'identité nationale est un concept censé réunir, qui se prête par conséquent à un traitement par le modernisme esthétique où les traits peuvent être stylisés et rendus dans un processus sinon abstrait, du moins dicté par des préoccupations formelles. En un certain sens, le formalisme crée une distance entre l'idée et le corps historicisé qui représente cette idée.

C'est en manipulant la notion de classicisme que Levinson s'empare, dans ses écrits, de La Argentina. Ce faisant, il lui attribue l'étiquette " classique » du simple fait qu'elle représente l'autre du classicisme (français), l'intégrant en cela à la catégorie universelle de l'altérité non occidentale. Pour ce faire, il fait de la danse ibérique une danse orientale :

Le mouvement de la danse orientale est concentrique - les genoux sont réunis et fléchis ; les bras dessinent des cercles et embrassent le corps ; tout s'assemble et converge vers le centre - alors que le mouvement de la danse en Europe occidentale (dont on trouve la plus parfaite expression dans la danse traditionnelle classique) fait exactement l'inverse - le corps de la danseuse étant en extension, bras et jambes tournés vers l'extérieur, le torse libéré et la poitrine projetée en avant ${ }^{17}$.

Par cette théorisation esthétique, Levinson engendre une sorte de dipôle, ou ce qu'il nomme une " antinomie " et une " antithèse ", composé de la danse européenne en opposition à l'autre danse, catégorie dans laquelle il intègre la " danseuse arabe " et la bayadère tout comme la danseuse espagnole, qu'il qualifie $\mathrm{d}^{\prime}$ " andalouse ». " Il semble 
clair - écrit Levinson - que les splendeurs et les misères, la gloire et la décadence de la danse espagnole se sont déterminées à la faveur de l'affrontement ininterrompu entre l'Orient et l'Occident, les ardeurs brûlantes du Maure et l'austérité de l'esprit castillan $^{18}$. " En somme, la danse met en évidence " l'interpénétration mutuelle des races, la nature duale du Sémite et de l'Arien ". Ici, Levinson ajoute l'influence juive à l'influence mauresque, afin de donner de la danse espagnole l'image d'un " éternel champ de bataille ${ }^{19}$ ". Et c' cest précisément là que sa formation classique vient en aide à la danseuse : "Elle a conservé l'ampleur de mouvement de la danseuse classique, car elle a reçu cette formation, modifiée par les principes espagnols ${ }^{20}$. " Levinson compare ces principes à un fluide qui circule dans ses veines et la fait presque souffrir. "Elle porte en elle le poison qui l'intoxique, mais aussi son antidote, l'extase et le maintien, la folie et la sagesse, la ménade et la muse ${ }^{21}$. " C'est en ce sens que la danseuse est un champ de bataille, et la danse une bataille où triomphe in fine l'influence européenne. La Argentina est classique du simple fait d'être l'autre du classicisme dans une lutte pour se dépasser elle-même.

Si c'est en ces termes que Levinson loue La Argentina, parvenue à un autre classicisme, Onís, lui-même espagnol, salue en elle une poète qui transmet sa propre vision de l'Espagne. Il n'allait pas de soi, pour Onís, que l'identité espagnole puisse être représentée sur scène, car un mouvement qui s'appuierait sur l'idée de types nationaux aboutirait à une caricature. C'est en revanche par leur utilisation du rythme que se distinguent, pour Onís, La Argentina et son art :

Pour comprendre quoi que ce soit d'espagnol, il nous faudrait en sentir l'animation par un rythme interne et original, et nous parviendrions ainsi à découvrir les éléments essentiels et permanents du rythme espagnol qui bat en chacun de ces éléments ${ }^{22}$.

La façon dont Onís conçoit l'identité nationale, provisoire et proprement personnelle, se passe, au contraire de ce que défend Levinson, de toute intervention de la forme ou de la mimêsis. Elle est, dans sa nature, plus gestuelle, et procède d'un savoir-faire ${ }^{23}$ qui s'appuie sur une démarche personnelle. Cette identité nationale s'incarne en outre de maintes façons, portées par maints interprètes, notamment parce que la forme par excellence de la danse espagnole est le solo, qu'Onís oppose en fait à l'identité d'exécution dont fait preuve le corps de ballet.

Si nous tentons de nous imaginer la forme la plus commune et typique de la danse anglo-saxonne, nous nous rappellerons une troupe de jeunes filles toutes semblables qui réalisent avec la précision et l'uniformité la plus grande les mêmes mouvements ; la danse espagnole, en revanche, nous l'imaginerions telle que nous la voyons avec La Argentina, une personne seule qui se meut avec la plus grande liberté et qui fait apparemment ce dont elle a envie. La danse anglo-saxonne, comme le sport, les affaires, la politique, la morale, la religion anglo-saxonnes, est essentiellement sociale ; la danse espagnole, comme toutes les manifestations de la culture espagnole, est essentiellement individuelle ${ }^{24}$.

Onís conçoit le nationalisme espagnol comme indivisible de l'individualité. Il est intéressant de noter que c'est du monde anglo-saxon, y plaçant implicitement la France, qu'il distingue l'Espagne, tandis que c'est de l'Europe que Levinson la différencie, pour la placer dans la catégorie " orientale ». Si l'on suit Onís, l'expression esthétique de l'identité nationale par le mouvement est en France fondamentalement sociale, tandis qu'en Espagne, elle est individuelle. La danse espagnole, en d'autres termes, n'est pas dictée par la forme, mais par le contenu gestuel individuel. Si, pour Levinson, la musique est d'une importance secondaire par rapport au corps dans la danse, la rythmicité est pour Onís la qualité qui crée le sentiment d'une communauté par lequel peut émerger 


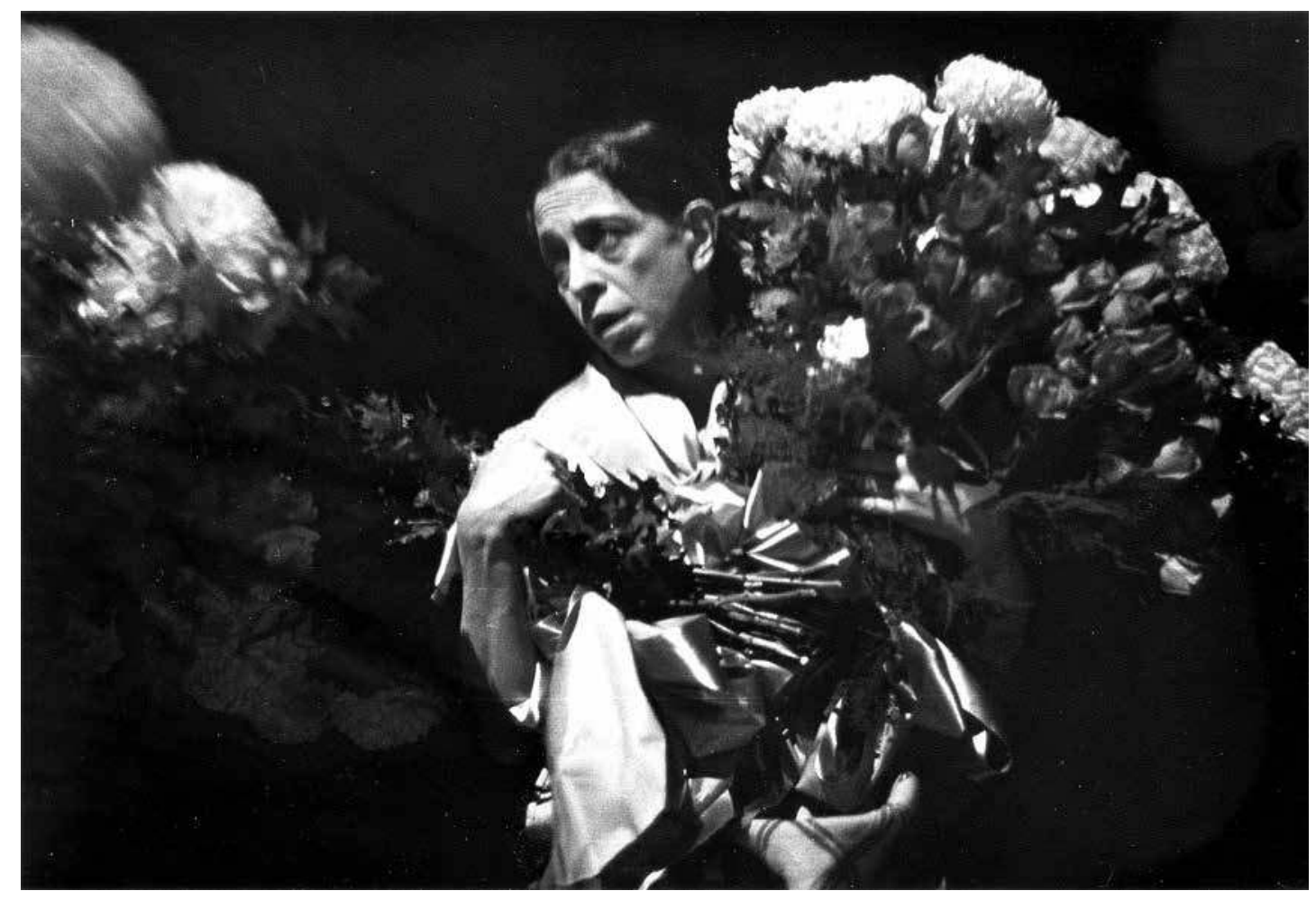

le concept d' " espagnol " en tant que tel dans la danse. C'est pour cette raison que la danse doit être une distraction ou un divertissement sans but déterminé, idée suggérée 4. Gordon Pollock, Antonia Mercé, La Argentina, Town Hall, New York City, vers 1935, New York Public Library, Dance division. par les réflexions de Valéry. Si la danse de La Argentina acquiert, en retravaillant des chorégraphies traditionnelles, une dimension classique dans ses représentations des types de personnages, c'est, pense Levinson, parce que son pouvoir de mimêsis est déterminé, lors de chaque numéro, par une maîtrise technique de la forme et de la nuance. Pour reprendre ses termes, l'expression individuelle de La Argentina se perfectionne par les améliorations successives portées à la nuance de son exécution afin d'affiner l'effet recherché.

Onís part de son analyse du rythme pour aboutir à l'idée que la marche, le pas, est bien l'acte fondamental de la danse espagnole par lequel peut être saisie son esthétique particulière : " La marche constitue par elle-même en Espagne un spectacle [porteur] de la plus grande émotion artistique ${ }^{25}$. " L'action humaine qu'est la marche autorise à comparer les peuples. Cette action est de l'ordre du geste, qui peut communiquer l'imaginaire d'un caractère national sans exprimer pour autant quelque organisation sociale que ce soit. Si la marche peut être analysée en termes formels - pour Onís, c'est la façon de lever le pied qui est cruciale et l'accent donné à ce geste, dont la qualité ne dépend pas des critères retenus par Levinson, en l'occurrence de l'opposition entre mouvement excentrique et concentrique.

Onís résume les qualités de la danse espagnole telle que l'incarne La Argentina depuis la perspective des qualités esthétiques dont fait montre sa danse, qui définissent aussi un caractère national : 
Individualité, liberté, élan vers le haut, gestes inattendus et contradictoires,

tragique et douceur, grâce et violence, tout cela, sujet à un rythme interne auquel

on revient toujours, comme dans les si libres chansons espagnoles, constitue

l'âme de l'Espagne, que nous voyons incarnée dans La Argentina lorsqu'elle danse ${ }^{26}$.

Levinson voyait danser La Argentina comme si elle se livrait combat à elle-même afin de libérer la possibilité de l'incarnation du caractère national, ce qui exigeait une expression classique, comprise comme la domination de l'esthétique sur la personnalité. Onís attribue quant à lui à cette expression des qualités éminemment personnelles, mais dépersonnalise aussi le processus symbolique en le comprenant comme ce qui transpire dans le corps plutôt que dans la personnalité, devenue champ de bataille entre Orient et Occident.

Dans son "Éloge », Lorca, qui qualifie l'art de la danseuse de personalísimo ( "personnalissime ", ou « infiniment personnel »), suit une logique similaire à celle d'Onís ${ }^{27}$. Pour le poète, qui a pu assister à des représentations de La Argentina à New York (fig. 4), sa danse est comme un "nouveau-né, inséparable [du] corps [de sa mère], un acte qui jamais plus ne pourra se répéter ". La singularité et la non-reproductibilité sont des qualités bien identifiables de la créativité moderniste, souvent évoquées à propos de la danse moderne nord-américaine des années 1930. Le succès de La Argentina à New York est une reconnaissance de sa place dans l'esprit moderne du temps, où personnalité, indigénéité et universalité avancent de pair. Son succès parisien semble avoir été plus dépendant de l'idée, du moins pour Levinson, qu'elle était un sujet divisé, un sujet qui devait surmonter son indigénéité afin d'atteindre à l'universalité.

Cette contribution a été traduite de I'anglais par François Boisivon. 


\section{Mark Franko}

Mark Franko est professeur de danse, titulaire de la chaire Laura H. Carnell à la Temple University de Philadelphie. Il est l'auteur de Choreographing Discourses: A Mark Franko Reader (Routledge, 2019) et a dirigé The Oxford Handbook of Dance and Reenactment (Oxford University Press, 2017). Il a été lauréat, en 2011, du Congress on Research in Dance Outstanding Scholarly Research Award et a obtenu, en 2018-2019, une bourse de la Fondation en la mémoire de John Simon Guggenheim pour The Fascist Turn in the Dance of Serge Lifar: Interwar French Ballet and the German Occupation (Oxford University Press, 2020). Son ouvrage La Danse comme texte : idéologies du corps baroque (1993) a été traduit en français en 2005 (par Sophie Renaut).

\section{NOTES}

1. Idoia Murga Castro, "Los Ballets Españoles de Antonia Mercé La Argentina ", dans Escenografía de la Danza en La Edad de Plata (1916-1936), Madrid, Consejo Superior de Investigaciónes Científicas, 2017, p. 139180. Je veux ici remercier Linda Murray et l'équipe du département de Danse de la bibliothèque des Arts de la scène du Lincoln Center à New York, pour leur aide précieuse qui m’a permis d'avoir accès aux ressources nécessaires à mes recherches durant la fermeture des bibliothèques consécutive à la pandémie.

2. Voir Idoia Murga Castro, «Embodying Spanishness: La Argentina and her Ballets Espagnols ", dans Conversations, vol. XXXIX, Dance Studies Association, 2019, p. 12-17.

3. La Argentina donna ses représentations au Philosophy Hall I'année même où Federico García Lorca, accueilli en résidence à la Columbia University, composa le recueil Poeta en Nueva York (Le Poète à New York). Lorca, après avoir vu sur scène La Argentina, écrivit "Elogio de Antonia Mercé, La Argentina », dont il sera reparlé à la fin de cet article.

4. Mark Franko, The Fascist Turn in the Dance of Serge Lifar: French Interwar Ballet and the German Occupation, New York, Oxford University Press, 2020.

5. Kenneth E. Silver, Esprit de Corps. The Art of the Parisian Avant-Garde and the First World War (19141925), Princeton, Princeton University Press, 1989.

6. Pour un aperçu biographique sur Onís, voir Howard Young, " Federico de Onís (1888-1966) ", dans Hispania, n 80 , mai 1997 , p. 268-270.

7. José Álvarez Junco, Dioces útiles. Naciones y nacionalismos, Barcelone, Galaxia Gutenberg, 2017, p. 174-175.

8. Valéry prononça cette allocution le 5 mars 1936 et elle fut publiée pour la première fois sous le titre "La Danse, Conférence de M. Paul Valéry », dans Conferencia, 30 année, ${ }^{\circ}$ XXII, $1^{\text {er }}$ novembre 1936. Elle a, depuis, été intégrée à la publication des œuvres de Valéry et est considérée comme l'un de ses textes importants sur la danse. Pour une analyse plus approfondie, voir mon article "The Dancing Gaze Across Cultures: Kazuo Ohno's Admiring La Argentina », dans Mark Franko (dir.), Choreographing Discourses: A Mark Franko Reader, Alessandra Nicifero (coll.), Londres, Routledge, 2019, p. 154-173.

9. Je préfère ne pas utiliser le terme d'« hispanité » [spanishness, employé par Idoia Murga Castro], qui nous prédispose à l'idée d'une essence ou d'un condensé, obscurcissant le fait que la danse survient à l'existence en tant que représentation matérielle en un lieu et un temps précis avant de pouvoir être considérée comme une abstraction visuelle ou comme une allégorie.

10. Dans ma récente étude consacrée à la réception par Levinson du danseur de ballet Serge Lifar, je m'intéresse au conflit entre le classique et le moderne et à son apaisement dans l'entre-deux-guerres, à la faveur de l'élaboration d'une théorie du ballet néo-classique. Sur le développement de l'idée de ballet néo-classique dans la France de l'entre-deux-guerres, voir " The Critical Reception of Serge Lifar (1929-1939) ", dans Franko, 2020, cité n. 4, p. 54-108.

11. Voir Javier Suárez-Pajares, "Historical Overview of the Bolero from its Beginnings to the Genesis of the Bolero School ", Aurelio de La Vega (trad. fr.), dans Javier Suárez-Pajares et Xoán M. Carreira (dir.), The Origins of the Bolero School. Studies in Dance History, vol. IV, nº 1, printemps 1993, p. 1-19.

12. Antonia Mercé, La Argentina, «El baile español (1917) ", réimpression dans Idoia Murga-Castro (dir.), Poetas de Cuerpo. La danza en la Edad de Plata, Madrid, Publicaciones de la Residencia de Estudiantes, 2017, p. 404 et suivantes.

13. Voir "Folklore and Parade from Jean Cocteau and Paul Valéry to André Varagnac: The Resurgence of French Folkloric Studies », dans Franko, 2020, cité n. 4, p. 129-179.

14. Cette racialisation de la dance rappelle le travail de I'historien de la danse Curt Sachs, qui fut le contemporain de Levinson.

15. La figure prédominante utilisée comme explication de la dualité de la représentation dansée est empruntée à La Naissance de la tragédie de Nietzsche (1872) où l'idée d'une forme est associée à Apollon, tandis que celle de l'énergie et de la perte du contrôle de soi l'est à Dionysos.

16. André Levinson, La Argentina. A Study in Spanish Dancing, Paris, Éditions des Chroniques du jour, 1928, p. 58.

17. Ibid., p. 35

18. Ibid., p. 34.

19. Ibid.

20. Ibid., p. 54.

21. Ibid., p. 78.

22. Federico de Onís, "Ritmo y Cultura », dans Angel del Río et al., Antonia Mercé, La Argentina, New York, Instituto de las Españas en los Estados Unidos, 1930, p. 42.

23. En français dans le texte. 
24. Onís, 1930, cité n. 22, p. 45.

25. Ibid., p. 47.

26. Ibid., p. 48.

27. Federico García Lorca, "Elogio de Antonia Mercé, La Argentina ", dans Federico y su mundo, Madrid, Alianza Tres, 1980, p. 469. 